\title{
Plasma Catecholamine in Spontaneously Hypertensive Rat Assayed by Double Isotope Method
}

\author{
Jun Hashida, Hiroshi Terashima, Kojin Kin, Tateo Tel, \\ Yasuo Izeki, Takeshi Mikami, Masashi Ono, \\ Takahisa Uchiyama, Takashi Krta, Masahiko Sato, \\ akira Murakami, Yoshiko Kobayashi, and Nagao Kajiwara
}

The enzymatic double isotope derivative assay and usual fluorescence assay were applied to the measurement of the catecholamines in plasma samples obtained from patients with essential hypertension and spontaneously hypertensive rats (SHR). The catecholamine concentrations measured by radioisotope method were correlative to those by fluorescence assay.

The plasma catecholamine concentrations by radioisotope assay in the 11 hypertensive patients $(\mathrm{NE}=0.12 \pm 0.05 \mu \mathrm{g} / \mathrm{L}, \mathrm{E}=0.06 \pm 0.03 \mu \mathrm{g} / \mathrm{L}[\mathrm{M} \pm \mathrm{SD}]$ ) were higher than those in 6 normal subjects $(\mathrm{NE}=0.09 \pm 0.007 \mu \mathrm{g} / \mathrm{L}, \mathrm{E}=$ $0.02 \pm 0.005 \mu \mathrm{g} / \mathrm{L})$.

Plasma norepinephrine concentrations were not correlative to the mean blood pressure in hypertensive patients.

The plasma catecholamine concentrations determined by radioisotope assay in $7 \mathrm{SHR}$ ( $\mathrm{NE}=0.28 \pm 0.09 \mu \mathrm{g} / \mathrm{L}, \mathrm{E}=0.04 \pm 0.026 \mu \mathrm{g} / \mathrm{L}[\mathrm{M} \pm \mathrm{SD}]$ ) were higher than those of $8 \mathrm{Wistar}$ rats $(\mathrm{NE}=0.20 \pm 0.10 \mu \mathrm{g} / \mathrm{L}, \mathrm{E}=0.03 \pm 0.002 \mu \mathrm{g} / \mathrm{L}$ $[\mathrm{M} \pm \mathrm{SD}]$ ).

Neither in SHR nor in hypertensive patients, plasma norepinephrine concentrations were correlative to the systolic blood pressure.

From the Second Department of Internal Medicine, Nihon University, School of Medicine, Tokyo. 